\title{
Metabolic engineering with multi-objective optimization of kinetic models
}

\author{
Alejandro F Villaverde ${ }^{\mathrm{a}, \mathrm{b}, \mathrm{c}}$, Sophia Bongard ${ }^{\mathrm{d}}$, Klaus Mauch ${ }^{\mathrm{d}}$, Eva \\ Balsa-Canto $^{a}$, Julio R Banga ${ }^{a}$ \\ ${ }^{a}$ Bioprocess Engineering Group, IIM-CSIC, Eduardo Cabello 6, 36208 Vigo, Spain \\ ${ }^{b}$ Centre of Biological Engineering, Universidade do Minho, Campus de Gualtar, \\ 4710-057 Braga, Portugal \\ ${ }^{c}$ Department of Systems and Control Engineering, Universidade de Vigo, Rua Maxwell, \\ 36310 Vigo, Spain \\ ${ }^{d}$ Insilico Biotechnology AG, Meitnerstraße 9, 70563 Stuttgart, Germany
}

\begin{abstract}
Kinetic models have a great potential for metabolic engineering applications. They can be used for testing which genetic and regulatory modifications can increase the production of metabolites of interest, while simultaneously monitoring other key functions of the host organism. This work presents a methodology for increasing productivity in biotechnological processes exploiting dynamic models. It uses multi-objective dynamic optimization to identify the combination of targets (enzymatic modifications) and the degree of up- or down-regulation that must be performed in order to optimize a set of pre-defined performance metrics subject to process constraints. The capabilities of the approach are demonstrated on a realistic and computationally challenging application: a large-scale metabolic model of Chinese Hamster Ovary cells $(\mathrm{CHO})$, which are used for antibody production in a fed-batch process. The proposed methodology manages to provide a sustained and robust growth in $\mathrm{CHO}$ cells, increasing productivity while simultaneously increasing biomass production, product titer, and keeping the concentrations of lactate and ammonia at low values. The approach presented here can be used for optimizing metabolic models by finding the best combination of targets and their optimal level of up/down-regulation. Furthermore, it can accommodate additional trade-offs and constraints with great flexibility.
\end{abstract}

Keywords: dynamic modelling, multi-objective optimization, large-scale, metabolic engineering, target identification, up/down-regulation 


\section{Introduction}

Model-based approaches can be used to improve biotechnological processes. In model-based metabolic engineering, mathematical models are used to guide the optimization of metabolic processes aiming at enhanced production of a certain compound. Model-based metabolic engineering techniques can be broadly classified into two categories, depending on the type of models used: static or kinetic.

Constraint-based methods such as flux balance analysis (FBA) (Varma and Palsson, 1994; Kauffman et al., 2003; Orth et al., 2010) use static models to calculate steady-state fluxes. These models focus on stoichiometry and do not include kinetic detail, and as a consequence they cannot predict how global metabolic behavior emerges from dynamic concentration changes of cellular components. Recent reviews of the state of the art in constraintbased modeling for metabolic engineering applications can be found in Long et al. (2015); King et al. (2015); Machado and Herrgard (2015).

Kinetic models, on the other hand, allow for more detailed predictions. They can be used for calculating the alterations needed to optimize quantities such as the flux or yield of compounds of interest, while keeping the other functions of the host organism to a minimal, but essential, level (Smallbone and Mendes, 2013; Almquist et al., 2014; Song et al., 2013; Link et al., 2014).

Early on, extensions of constraint-based methods were developed with the aim of increasing the accuracy of predictions in cases where dynamic adaptations play an important role. Dynamic Flux Balance Analysis (dFBA) is an extension of FBA that takes into account the rate of change of flux constraints, which has been shown to improve predictions of the dynamics of diauxic growth (Mahadevan et al., 2002). In related applications, Henson and coworkers used dFBA for optimizing ethanol production both in $S$. cerevisiae fermentation (Hjersted and Henson, 2006) and in a batch co-culture of two yeasts (Hanly et al., 2013). More recently, the DySScO (Dynamic strain scanning optimization) strategy (Zhuang et al., 2013) has integrated dFBA with strain design algorithms, allowing optimizing not only yield but also product titer and productivity and taking into account economic considerations such as the price difference between product and feedstock.

Furthermore, in recent years a number of approaches have been presented for exploiting kinetic models in metabolic engineering applications. Nikolaev 
proposed a mixed-integer non-linear programming method for optimizing the enzyme levels and enzyme regulatory structure of metabolic models (Nikolaev, 2010) and applied it to a well-known kinetic reconstruction of E. coli (Chassagnole et al., 2002). Nolan and Lee presented a kinetic model of CHO cell metabolism (Nolan and Lee, 2011) and used it for metabolic engineering purposes (Nolan and Lee, 2012). They defined a set of possible modifications of certain process variables and gene knockdowns, allowing for a set of fixed levels of enzyme down-regulation. After performing the resulting simulations and selecting the feasible solutions, they obtained an ordered list of the best ones. Chowdhury and coworkers presented an optimization-based strain design framework, k-OptForce (Chowdhury et al., 2014), which is suitable for stoichiometric-based models for which mechanistic kinetic details are known for some equations. The methodology was applied to a kinetic model of $E$. coli to improve succinate yield (Khodayari et al., 2014); interestingly, however, this study also showed that predictions made by kinetic models may be worse than those obtained by FBA if the phenotype is significantly different.

Additional degrees of freedom can be achieved by implementing control strategies that change during the time course of the bioprocess, in response to changes in the process conditions (Venayak et al., 2015); for example, via dynamic optimal control of enzyme levels (Brockman and Prather, 2015). Recent applications of this strategy include, for example, the dynamic regulation of malonyl-CoA pools for improving fatty acid production (Xu et al., 2014).

Despite many significant advances, the use of realistic large-scale dynamic models is still hindered by a number of reasons: there are few of those models available, the experimental data required for their calibration is scarce, the associated optimization problems are computationally hard (Villaverde et al., 2015b), and there is usually large uncertainty regarding the detailed characterization of enzyme kinetics and enzyme regulation (Chakrabarti et al., 2013). For these reasons the use of kinetic models is not widespread in the biotechnology industry yet, although recent reviews strongly advocate for their exploitation (Almquist et al., 2014; Song et al., 2013; Link et al., 2014). To make this vision come true, new methodologies will be needed to overcome the associated difficulties.

With this aim we present here an approach that includes several contributions. The proposed methodology uses dynamic optimization to identify the combination of targets (i.e. enzymatic modifications) and determines the degree of up- or down-regulation that must be performed on them. It does 
so by applying a multi-objective optimization approach to obtain a Pareto front of solutions, from which the preferred trade-off between improvement in productivity-or, more generally, in any objective defined as a dynamic function of the system variables-and intervention effort can be selected. The control vector parameterization approach together with state of the art optimizers are used to solve the dynamic optimization problem. Furthermore, the method can accommodate additional requirements, such as keeping the concentrations of some metabolites within certain bounds throughout the process.

We demonstrate the capabilities of this approach by applying it to a challenging problem, the optimization of Chinese Hamster Ovary (CHO) cells for antibody production. Optimization of $\mathrm{CHO}$ strains is taking on greater significance in the industry. The emphasis is put on obtaining strains with robust growth and high metabolic product formation. However, the process of identifying robust targets is more challenging for $\mathrm{CHO}$ cells than for other hosts such as E. coli or yeast cells, due to their sensitive nature. Furthermore, product quality plays a key role since products from $\mathrm{CHO}$ cells are often used for the application in the human body (e.g. biologics). An in silico approach offers the possibility to remove several obstacles at once by generating a comprehensive kinetic model of the CHO metabolism, calibrating the $\mathrm{CHO}$ model to real process conditions, and optimizing the cell such that gene expression levels lead to improvement of multiple targets.

As has been already mentioned, kinetic models of $\mathrm{CHO}$ cells have been used for metabolic engineering purposes before (Nolan and Lee, 2012). However, in Nolan and Lee (2012) only gene knockdown was considered, and the allowed levels of enzyme down-regulation were fixed: either the nominal value or its $20 \%$ could be chosen, but no intermediate values. Furthermore, the solution space was explored by carrying out many simulations with different combinations of modifications, without applying optimization techniques. In contrast, here we allow for down- and up-regulation, explore the continuous range of values, and apply state of the art optimization algorithms to make sure that the solution space is thoroughly and efficiently explored. We also use a new, more complete CHO cells model. In several ways the approach presented here has more similarities with the one by Nikolaev (2010). However, compared to that work, we present key contributions: the ability to accommodate both end-point (final time) and path (throughout process duration) constraints with great flexibility, and its dynamic optimization formulation which allows designing time-varying inputs in a natural way. 


\section{Methods}

A graphical overview of the methodology is shown in Figure 1. A detailed description is provided in the following subsections.

\subsection{Objectives for bioprocess optimization}

We begin the presentation of the methodology by defining some terms frequently used in bioprocess engineering, and discussing the implications that the choice of the objective function has in the optimization outcomes.

Yield, $Y_{i j}$, is the ratio between the produced amount (mass) of a target metabolite $j$ and the consumed amount of a substrate $i$, that is:

$$
Y_{i j}=\frac{m_{j}^{p}}{m_{i}^{c}}
$$

The productivity is the industrial measure for the economic viability of a bioprocess. It is defined as the rate at which the target metabolites are produced. Here we focus on the specific productivity, which is the cell specific production rate $[\mathrm{g} /(\mathrm{gDW} \cdot \mathrm{h})]$. It is a measure of the performance of the cell to produce the desired product. The specific productivity multiplied with the cell concentration in the fermenter is the so called volumetric productivity $[\mathrm{g} /(\mathrm{l} \cdot \mathrm{h})]$. It represents a performance measure at the reactor level (Song et al., 2013).

Ideally, bioprocess optimization should aim at maximizing yield and productivity simultaneously. However, it is important to realize that maximizing yield does not necessarily imply maximizing productivity, because increasing product yield often leads to impaired growth of the microorganism (Song et al., 2013).

These are definitions typically found in the literature. However, some variants of them can also be used. For example, it should be taken into account that rate is an instantaneous quantity. Therefore, in a fed-batch process where the rate is not constant, it makes sense to maximize the integral of the rate, in which case the (volumetric) productivity would have units of concentration, not of rate (concentration/time). Note that if the reactor volume is constant, it is equivalent to speak of concentrations and masses. Maximizing the integral of the rate tends to maximize the total amount of substrate consumed during the process, instead of the instantaneous substrate consumption rate. 
In the present work we choose to maximize a productivity term defined as the integral of the rate of production of the target metabolite,

$$
P=\int_{t_{0}}^{t_{f}} r_{j}(t) d t
$$

where $r_{j}(t)$ is the instantaneous production rate $[\mu \mathrm{mol} /(\mathrm{gDW} \cdot \mathrm{h})]$ of the metabolite $j$, which in the case study used in this work is antibody formation rate, and $P$ has units $[\mu \mathrm{mol} / \mathrm{gDW}]$.

Finally, we note that it is also possible to perform a more detailed economic analysis of the process by including other factors, such as material costs and operation costs. The resulting benefit can then be expressed as the return on investment (ROI), which is defined as the net profit obtained divided by the cost of investment.

\subsection{Problem formulation: optimization of kinetic models for metabolic en- gineering}

The general problem addressed here is formulated as a dynamic optimization, where the objective is to find the decision variables which optimize a pre-defined performance index subject to the dynamic constraints and other possible bounds.

Mathematically, this is formulated as follows:

$$
\min _{v, u(t), t_{f}} J(x, v, u)
$$

subject to the following constraints:

$$
\begin{gathered}
\frac{d x(t)}{d t}=f(x(t), v, u(t), t, p) \\
x\left(t_{0}\right)=x_{0} \\
h_{e q}(x(t), v, u(t))=0 \\
h_{i n}(x(t), v, u(t)) \leq 0 \\
p h_{e q}\left(x\left(t_{i}\right), v, u\left(t_{i}\right)\right)=0 \\
p h_{i n}\left(x\left(t_{i}\right), v, u\left(t_{i}\right)\right) \leq 0 \\
v^{L} \leq v \leq v^{U} \\
u^{L} \leq u \leq u^{U}
\end{gathered}
$$


where $J(x, v, u)$ is the performance index to maximize, $t$ is the time variable, $x(t)$ is the vector of state variables with initial conditions $x_{0}, v$ is the vector of time-invariant decision variables, $u(t)$ is the vector of time-varying decision variables ( $v$ and $u$ are also called controls), $f$ is the set of differential and algebraic equality constraints describing the system dynamics (that is, the nonlinear system model), $p$ is the vector of model parameters (constants), $h_{e q}$ and $h_{i n}$ are equality and inequality path constraints that express additional requirements for the system performance, $p h_{e q}$ and $p h_{i n}$ are equality and inequality point constraints that must be verified at a given time (such as the final time, $t_{f}$ ), and $v^{L}, v^{U}, u^{L}$, and $u^{U}$ are lower and upper bounds for the vectors of decision variables $v$ and $u$.

The equations above define the general formulation of a dynamic optimization problem. For the case of a metabolic engineering application as the one considered here, they correspond to the following concepts:

- The objective function to optimize, $J$, is defined as the integral of the formation rate of antibody, as in equation (2).

- The decision variables $v$ are the enzyme levels. In the present application they are kept constant during the time course of the operation, and no time-varying decision variables $u(t)$ are considered. However, the methodology presented here can also be applied to the case of variables that vary in time-that is, control profiles consisting of series of steps, pulses or ramps can be taken into consideration.

- Apart from the differential equations defining the model dynamics, $f$, the additional constraints included in the formulation are some realistic lower and upper bounds for the state variables. In the application reported here we impose: (i) lower bounds on some concentrations as end-point constraints, $p h_{i n}$, that is, conditions that are only checked at the end of the time courses (to ensure that some key metabolites are not exhausted during the process); and (ii) lower and upper bounds for other quantities as path constraints, $h_{i n}$, that is, conditions that must be satisfied at all times (to ensure that some concentrations are always kept below or above some desired reference level). The choice of the particular type of constraints is application-dependent and should be made with attention to the process characteristics. The methodology presented here is sufficiently general to accommodate any combination of constraints. 
Using a numerical approach named control vector parametrization (CVP) (Vassiliadis, 1993) the original dynamic optimization problem is split into two sub-problems: an outer non-linear programming problem (NLP), and an inner initial value problem (IVP). The outer problem finds the optimal decision variables, that is, it optimizes the enzyme levels. The inner problem integrates the system dynamics. This approach has been recently shown to be able to predict activation in metabolic pathways (de Hijas-Liste et al., 2014).

Note that, in general, the outer problem could be formulated as a mixedinteger nonlinear programming problem (MINLP), where the aim would be to find (i) the set of decision variables that should be modified (discrete problem) and (ii) their optimal values (continuous problem). Instead, to facilitate the implementation, the outer optimization problem is solved here as a NLP, which is a relaxation of the more general MINLP. That is, we solve a continuous optimization problem where all the decision variables are in principle allowed to depart from the reference values. Not specifying a bound on the number of possible modifications would tend to lead to solutions where all the possible decision variables are modified. Hence to reduce the number of modified decision variables to a manageable quantity we adopt a multi-objective optimization approach using the $\epsilon$-constraint method, whose aim is to add a penalty to the modification effort. It is described in detail in the following subsection.

We note that, since the models we are handling are non-linear and largescale, the outer optimization problem is multimodal and very challenging to solve. Therefore it is necessary to use proper global optimization methods (Banga et al., 2005). Specifically, we have used here the eSS optimization metaheuristic (Egea et al., 2010) implemented in the AMIGO toolbox (BalsaCanto and Banga, 2011). Furthermore, since eSS is a hybrid method that can make use of local methods to accelerate performance, we have used it in combination with SOLNP (Yinyu, 1990) as a local solver.

Importantly, we remark that the methodology allows for the precise tuning of the optimal enzyme levels $v$, that is, the exact amount of up- and down-regulation that must be performed to optimize the performance. This is a different and more powerful tool than simply finding the optimal knockdowns, or even choosing the optimal level among a pre-specified set. 


\subsection{Multi-objective optimization}

The problem considered in this work involves a trade-off between two conflicting objectives: on the one hand the aim is to maximize productivity, while on the other hand we wish to keep the number of interventions (i.e. enzymatic modifications) as small as possible. To address this problem properly we modify the formulation presented in the preceding subsection, and consider the performance index in equation (3) as a set of two cost functions:

$$
J(x, v)=\left[\begin{array}{l}
J_{1}(x, v) \\
J_{2}(x, v)
\end{array}\right]
$$

where the first objective to be optimized $\left(J_{1}\right)$ is the productivity defined in equation (2). The second objective $\left(J_{2}\right)$ is the modification effort,

$$
J_{2}=\left\|v-v_{0}\right\|
$$

where $v$ is the vector of decision variables (enzyme levels) and $v_{0}$ is the reference vector of decision variables, that is, the enzymatic levels of the wild type cell. We choose as norm the absolute value,

$$
J_{2}=\left\|v-v_{0}\right\|=\sum_{i=1}^{n}\left|v(i)-v_{0}(i)\right|
$$

where $n$ is the vector length and $v(i)$ is its $i^{t h}$ element.

To appropriately address the goal of optimizing two conflicting objectives simultaneously we adopt a multicriteria optimization approach. The outcome of a multicriteria or multiobjective optimization (MOO) procedure is the socalled Pareto front of non-dominated solutions. Each of the solutions in the Pareto front represents an optimal trade-off between both objectives, $J_{1}$ and $J_{2}$. Thus, it is not possible to improve one of them without worsening the other.

We obtain the Pareto front using the $\epsilon$-constraint method (Haimes et al., 1971). This approach is conceptually simple: it consists of performing several optimizations, each of which optimizes one objective (i.e. maximize productivity, $J_{1}$ ) while using the remaining objective as an inequality constraint (i.e. keep the modification effort, $J_{2}$, below a maximum value). Setting $J_{2}$ to a different value in every optimization (or in other words, setting a different upper bound for the inequality constraint) leads to different solutions, each of which represents the maximum productivity achievable for the allowed 
modification effort. The solutions found in this way constitute the Pareto front. More details about the application of this method to the CHO cells model are given in section 3.2.

In our formulation, all the decision variables $v$ (enzyme levels) are in principle allowed to be modified, that is, the number of interventions is not selected a priori. Since the $\epsilon$ constraint imposes an upper limit on the overall deviation from the reference levels, the total amount of modification becomes a limited resource. Hence the optimization algorithm will concentrate on modifying the enzymes which exert a greater influence on the productivity, while leaving the less influential unmodified. This will result in few enzyme levels with significant modifications, along with many others with very small modifications (the proportion of enzymes in one or other group will depend on the chosen value of the $\epsilon$ constraint). Due to this fact the whole optimization procedure is carried out in two steps:

1. Calculation of the Pareto front and selection of the preferred trade-off solution: perform $m$ optimizations corresponding to different levels of $\epsilon$, i.e. $\epsilon=\left[\epsilon_{1}, \epsilon_{2}, \ldots, \epsilon_{m}\right]$. This results in a Pareto front consisting of a set of $m$ solutions, each of which corresponds to an optimal trade-off between productivity enhancement and modification effort. From the Pareto front we choose our preferred solution, taking into account the number of enzymatic modifications that is reasonable to perform.

2. Refinement of the preferred solution: take the solution selected in the previous step and reset all the enzyme levels whose values were not significantly altered during optimization to their reference values. This will usually result in a solution whose performance is only slightly worse than the one found in the optimizations. This new solution-to which we refer here as "fixed" solution for convenience-is then taken as the starting point for a subsequent refinement through a new optimization, in which the only decision variables allowed to change are the ones that underwent significant modifications in the previous step.

The outcome of this procedure is a model which optimizes productivity while satisfying other constraints, with an adjustable number of interventions.

Finally, we note that unlike other procedures such as the weighted sum approach, the $\epsilon$-constraint method is able to identify a nonconvex boundary. An example of $\mathrm{MOO}$ of biological networks using the $\epsilon$-constraint method (without involving integration of kinetic models) can be found in (Sendin et al., 2010). 


\section{Results and Discussion}

\subsection{Case study: CHO cell model for antibody production}

We applied the proposed dynamic optimization approach to optimize antibody production in Chinese Hamster Ovary cells (CHO). To this end, we extended a recently presented compartmented large-scale kinetic model of $\mathrm{CHO}$ metabolism (Villaverde et al., 2015a), which is included in the BioPreDynbench collection (Villaverde et al., 2015b). We took the biomass and antibody composition and experimental data from Nolan and Lee (2011). To be able to describe the phenotype, we added the uptake, consumption, formation and degradation of multiple amino acids in the model. The ODE model comprises now reactions from the central carbon metabolism as well as elements from the amino acids metabolism, mitochondrial respiration, and generation of unwanted by-products (lactate and ammonia). A diagram of the network is shown in Figure 2. It involves 54 balanced compounds and 45 reactions (cf. supplementary table $\mathrm{S} 1$ ). All reactions are modeled with linlog kinetics, with exception of biomass and antibody formation, for which irreversible Michaelis-Menten kinetics are chosen to avoid reversibility due to accumulating product effects. The model has a total of 209 kinetic parameters and 44 possible enzymatic modifications. The model simulates a bioprocess consisting of two steps: first, a batch process of 70 hours, followed by a fed-batch process lasting for additional 160 hours, during which glucose, cysteine, serine and glutamine are fed.

In order to speed-up the computations, the model dynamics were implemented in FORTRAN, and a Matlab file was created for interfacing the model with the AMIGO Matlab toolbox (Balsa-Canto and Banga, 2011). Integration was performed with the RADAU5 solver (Hairer and Wanner, 2005) enforcing tight integration tolerances (relative: $10^{-9}$, absolute: $10^{-12}$ ). Using the scatter search optimization metaheuristic included in the AMIGO toolbox, we calibrated the model with experimental data taken from (Nolan and Lee, 2011), which consists of 11 time points for each of the 13 measured metabolites. We managed to obtain a good fit-shown in Figure 3-which is qualitatively similar to the one reported in the original publication.

We note that, since the data set used for model calibration is relatively small compared to the number of unknown parameters, several parameter vectors can lead to similarly good fits. This fact, which denotes poor practical identifiability, does not necessarily prevent models from making useful predictions (Cedersund, 2012), and it can be addressed with an ensemble 
modelling approach such as the one in (Villaverde et al., 2015a), among other possibilities. Future work will involve more detailed analyses of the uncertainty in model calibration and its consequences for model predictions.

\subsection{Multi-objective optimization of $\mathrm{CHO}$ cells}

After calibrating the model we applied the procedure presented in the Methods section. Recall that in this MOO problem we consider two objectives: achieving maximal productivity with a minimum of interventions. With the $\epsilon$-constraint method this means maximizing the first objective $J_{1}$, which is the productivity defined in equation (2), while using the second objective $J_{2}$ as an inequality constraint. The second objective $J_{2}$ is the modification effort defined in equations (13) and (14). In this application the reference decision variables $v_{0}$ are normalized to a unit value, and the elements of the vector $v$ are allowed to change during optimization in the range $[0.2,2]$. This application does not require the definition of equality constraints as in equations (6)-(8), although they could be included if necessary. Regarding inequality constraints, we define several end-point constraints as in equation (9), to enforce that:

1. Product titer (i.e. antibody concentration at final time) and biomass are higher in the optimal cell than in the wild type cell. This constraint is required because optimizing productivity may lead to reduced production of biomass, and increased productivity does not always correlate directly to increased product titer.

2. The concentrations of certain metabolites at final time are kept above zero, to ensure that they are not exhausted during the process.

Finally, we also define some path constraints which must be fulfilled during the whole time course of the process:

1. The concentrations of ammonia and lactate are always kept below a reference value.

2. The energy charge of the cell is always kept above a reference level.

After defining the problem in this way, we optimized the model for several values of $\epsilon$ and obtained the Pareto front shown in Figure 4. As expected, as the number of allowed modifications increases, so does the achievable objective (i.e. productivity). However, we are interested in a solution that can be obtained with a reasonable number of enzymatic modifications, typically 4 or 
5. Hence from the set of obtained solutions we selected the one corresponding to $\epsilon=2$, which presents only 4 significant modifications and manages to improve productivity while satisfying the remaining constraints, as desired. By "significant" we refer to changes larger than 10\% with respect to the reference value of one, that is, values in the range $[0.9,1.1]$. The identified modifications and their optimal values are shown on Figure 4 and in column (II) of Table 1.

Note that the value of $\epsilon$ that leads to a particular number of significant modifications (in this case, four) is not known a priori, because it depends on the way that the "modification budget" represented by $\epsilon$ is spent. Recall that $\epsilon$ is not the number of modifications, but the sum of the deviations between the reference enzyme levels and the modified enzyme levels. Thus, a value of $\epsilon=2$ could also lead to only 3 significant modifications instead of 4 , for example.

\subsection{Refinement of the initial solution}

The solution reported in the previous subsection contained a large number of decision variables which are only slightly modified, that is, their values lie in the range $[0.9,1.1]$. After fixing their values to the initial reference value of one, we define a new optimization where we only allow to change the four enzyme levels listed above. We take their optimized values as the initial point for a subsequent optimization to refine this solution, arriving at the set of values shown in column (III) of Table 1.

The most important process outcomes obtained with these new levels are shown in Figure 5, from which it is clear that the optimization procedure succeeds in improving the original system. It should be noted that the quantities in the upper row (productivity, antibody concentration, and amount of biomass) should be increased with respect to the wild type, while the quantities in the lower row (concentrations of lactate and ammonia) should remain low. The desired outputs are achieved in both cases; notably, the optimized model avoids the peaks in ammonia and lactate concentration, while at the same time providing a sustained and robust growth.

\subsection{Biological interpretation of the solution}

The optimal enzyme modifications found were: glutamate dehydrogenase, glutamate-ammonia ligase (also known as glutamine synthetase), glucose phosphate isomerase, and mitochondrial glutamate carrier, Slc24a18/Gc2. 
One of these modifications is actually quite common: a knockout or knockdown of the glutamine synthetase, as predicted in our approach, is found in many high-producer CHO cell mutants (Fan et al., 2012, 2013). Ammonia inhibits the growth of CHO cells (Zhang et al., 2006) and in mammalian cells the capacity for ammonia removal is higher in the absence than in the presence of glutamine (Choi et al., 2000). Therefore a glutamine synthetase deficiency results in lower ammonia level, and has a positive effect on growth (Zhang et al., 2006). On the other hand, the proposed downregulation of glucose-6-phosphate isomerase leads to decreased glycolytic fluxes resulting in a reduction of lactate. Experimentally the loss of glucose-6-phosphate isomerase activity resulted in a temperature sensitivity and severe reduction in the synthesis of glycerolipids due to a reduction in phosphatidate phosphatase (Haller et al., 2011). Furthermore, a knockdown reduces the carbon supply which is needed to cover energy needs of the cell. Hence, at first sight the knockdown modification of the glucose-6-phosphate isomerase proposed by our method is counterintuitive. However, the predicted glutamate dehydrogenase overexpression helps to solve the energy dilemma. The glutamate dehydrogenase pathway is usually preferred when the energy demand is not fulfilled because of glucose exhaustion (Altamirano et al., 2013). The glutamate dehydrogenase pathway is also known to be more efficient than the transaminase pathway (Altamirano et al., 2013). Furthermore, the overexpression of the mitochondrial glutamate carrier Slc24a18/Gc2 increases the import of glutamate into the mitochondrion. Thus, this combination of targets manages to reduce lactate production (via glucose-6-phosphate isomerase knockdown), on the one hand, and on the other hand it fulfils the modified energetic needs (Slc24a18/Gc2 and glutamate dehydrogenase overexpression).

\section{Conclusions}

We have presented a methodology for increasing productivity in biotechnological processes exploiting dynamic models. The method identifies the combination of targets (enzymatic modifications) and determines the exact degree of up- or down-regulation that must be performed. This allows for more flexibility than simply finding the optimal knock-outs, or even finding the optimal regulation levels from a set of preset values. Furthermore, the procedure can accommodate additional requirements, such as the need of keeping the concentrations of some metabolites within certain bounds. Im- 
portantly, the multi-objective optimization approach that lies at the core of the methodology applies a so-called $\epsilon$-constraint technique, which generates a Pareto front of nondominated solutions. Having a set of optimal solutions instead of a single one allows to select the preferred trade-off between improvement in productivity and number of required interventions.

We have illustrated the capabilities of the approach applying it to a largescale metabolic model of Chinese Hamster Ovary cells (CHO), which are used for antibody production in a fed-batch process. Optimization of $\mathrm{CHO}$ strains, with the double aim of achieving robust growth and high metabolic product formation, is of great importance in the biotechnology industry, but the sensitive nature of $\mathrm{CHO}$ cells makes it challenging to obtain robust predictions. Here we showed that the proposed methodology manages to provide a sustained and robust growth in $\mathrm{CHO}$ cells, increasing productivity while simultaneously increasing biomass production, product titer, and keeping the concentrations of lactate and ammonia at low values.

We noticed that keeping the process variables fixed for the optimized strain limits possible improvements, since a new strain phenotype has altered feeding requirements. Therefore, future work will involve exploring the additional improvements that can be obtained by including the feed composition and volume as a time-varying decision variable, as well as the direct solution of the resulting mixed-integer dynamic optimization problem. We expect that the additional flexibility attained in this way will lead to substantial gains in productivity.

\section{Acknowledgments}

This work was supported by the EU project "BioPreDyn" (EC FP7KBBE-2011-5, grant number 289434) and MINECO project SYNBIOFACTORY (ref. DPI2014-55276-C5-2-R). Alejandro F Villaverde acknowledges support from the I2C postdoctoral programme funded by Xunta de Galicia (ref. ED481B2014/133-0). We would like to thank Joachim Schmid and Dirk Müller for their helpful comments.

\section{Appendix A. Supplementary data}

Supplementary data associated with this article can be found in the online version. The optimization software used in the calculations is available from the authors upon request. 


\section{References}

Almquist, J., Cvijovic, M., Hatzimanikatis, V., Nielsen, J., Jirstrand, M., 2014. Kinetic models in industrial biotechnology-improving cell factory performance. Metab Eng 24, 38-60.

Altamirano, C., Berrios, J., Vergara, M., Becerra, S., 2013. Advances in improving mammalian cells metabolism for recombinant protein production. Electronic Journal of Biotechnology 16 (3), 10-10.

Balsa-Canto, E., Banga, J. R., 2011. Amigo, a toolbox for advanced model identification in systems biology using global optimization. Bioinformatics 27 (16), 2311-2313.

Banga, J. R., Balsa-Canto, E., Moles, C. G., Alonso, A. A., 2005. Dynamic optimization of bioprocesses: Efficient and robust numerical strategies. Journal of Biotechnology 117 (4), 407-419.

Brockman, I. M., Prather, K. L., 2015. Dynamic metabolic engineering: New strategies for developing responsive cell factories. Biotechnology journal.

Cedersund, G., 2012. Conclusions via unique predictions obtained despite unidentifiability-new definitions and a general method. FEBS J 279 (18), 3513-3527.

Chakrabarti, A., Miskovic, L., Soh, K. C., Hatzimanikatis, V., 2013. Towards kinetic modeling of genome-scale metabolic networks without sacrificing stoichiometric, thermodynamic and physiological constraints. Biotechnology journal 8 (9), 1043-1057.

Chassagnole, C., Noisommit-Rizzi, N., Schmid, J. W., Mauch, K., Reuss, M., 2002. Dynamic modeling of the central carbon metabolism of escherichia coli. Biotechnol Bioeng 79 (1), 53-73.

Choi, Y. S., Lee, D.-Y., Kim, I. Y., Kang, S., Ahn, K., Kim, H. J., Jeong, Y. H., Chun, G.-T., Park, J. K., Kim, I.-H., 2000. Ammonia removal using hepatoma cells in mammalian cell cultures. Biotechnology progress 16 (5), 760-768.

Chowdhury, A., Zomorrodi, A. R., Maranas, C. D., 2014. k-optforce: integrating kinetics with flux balance analysis for strain design. PLoS computational biology 10 (2), e1003487. 
de Hijas-Liste, G. M., Klipp, E., Balsa-Canto, E., Banga, J. R., 2014. Global dynamic optimization approach to predict activation in metabolic pathways. BMC systems biology 8 (1), 1 .

Egea, J. A., Martí, R., Banga, J. R., 2010. An evolutionary method for complex-process optimization. Comput Oper Res 37 (2), 315-324.

Fan, L., Kadura, I., Krebs, L. E., Hatfield, C. C., Shaw, M. M., Frye, C. C., 2012. Improving the efficiency of cho cell line generation using glutamine synthetase gene knockout cells. Biotechnology and bioengineering 109 (4), 1007-1015.

Fan, L., Kadura, I., Krebs, L. E., Larson, J. L., Bowden, D. M., Frye, C. C., 2013. Development of a highly-efficient cho cell line generation system with engineered sv40e promoter. Journal of biotechnology 168 (4), 652-658.

Haimes, Y. Y., Ladson, L., Wismer, D. A., 1971. Bicriterion formulation of problems of integrated system identification and system optimization. IEEE Transactions on Systems Man and Cybernetics 1 (3), 296.

Hairer, E., Wanner, G., 2005. Solving ordinary differential equations ii. stiff and differential-algebraic problems.

Haller, J. F., Krawczyk, S. A., Gostilovitch, L., Corkey, B. E., Zoeller, R. A., 2011. Glucose-6-phosphate isomerase deficiency results in mtor activation, failed translocation of lipin $1 \alpha$ to the nucleus and hypersensitivity to glucose: Implications for the inherited glycolytic disease. Biochimica et Biophysica Acta (BBA)-Molecular Basis of Disease 1812 (11), 1393-1402.

Hanly, T. J., Henson, M. A., et al., 2013. Dynamic metabolic modeling of a microaerobic yeast co-culture: predicting and optimizing ethanol production from glucose/xylose mixtures. Biotechnol. Biofuels 6 (1), 44.

Hjersted, J. L., Henson, M. A., 2006. Optimization of fed-batch saccharomyces cerevisiae fermentation using dynamic flux balance models. Biotechnology progress 22 (5), 1239-1248.

Kauffman, K. J., Prakash, P., Edwards, J. S., 2003. Advances in flux balance analysis. Curr Opin Biotechnol 14 (5), 491-496. 
Khodayari, A., Chowdhury, A., Maranas, C. D., 2014. Succinate overproduction: A case study of computational strain design using a comprehensive escherichia coli kinetic model. Frontiers in bioengineering and biotechnology 2 .

King, Z. A., Lloyd, C. J., Feist, A. M., Palsson, B. O., 2015. Next-generation genome-scale models for metabolic engineering. Current opinion in biotechnology 35, 23-29.

Link, H., Christodoulou, D., Sauer, U., 2014. Advancing metabolic models with kinetic information. Curr Opin Biotechnol 29, 8-14.

Long, M. R., Ong, W. K., Reed, J. L., 2015. Computational methods in metabolic engineering for strain design. Current opinion in biotechnology 34, 135-141.

Machado, D., Herrgard, M., 2015. Co-evolution of strain design methods based on flux balance and elementary mode analysis. Metabolic Engineering Communications.

Mahadevan, R., Edwards, J. S., Doyle, F. J., 2002. Dynamic flux balance analysis of diauxic growth in escherichia coli. Biophysical journal 83 (3), 1331-1340.

Nikolaev, E. V., 2010. The elucidation of metabolic pathways and their improvements using stable optimization of large-scale kinetic models of cellular systems. Metabolic Engineering 12 (1), 26-38.

Nolan, R. P., Lee, K., 2011. Dynamic model of cho cell metabolism. Metabolic engineering 13 (1), 108-124.

Nolan, R. P., Lee, K., 2012. Dynamic model for cho cell engineering. Journal of biotechnology 158 (1), 24-33.

Orth, J. D., Thiele, I., Palsson, B. Ø., 2010. What is flux balance analysis? Nature biotechnology 28 (3), 245-248.

Sendin, J., Exler, O., Banga, J. R., 2010. Multi-objective mixed integer strategy for the optimisation of biological networks. IET systems biology 4 (3), 236-248. 
Smallbone, K., Mendes, P., 2013. Large-scale metabolic models: From reconstruction to differential equations. Industrial Biotech 9 (4), 179-184.

Song, H.-S., DeVilbiss, F., Ramkrishna, D., 2013. Modeling metabolic systems: the need for dynamics. Curr Opin Chem Eng 2 (4), 373-382.

Varma, A., Palsson, B. O., 1994. Metabolic flux balancing: Basic concepts, scientific and practical use. Bio/technology 12.

Vassiliadis, V., 1993. Computational solution of dynamic optimization problems with general differential-algebraic constraints. Ph.D. thesis, Imperial College London (University of London).

Venayak, N., Anesiadis, N., Cluett, W. R., Mahadevan, R., 2015. Engineering metabolism through dynamic control. Current opinion in biotechnology 34, $142-152$.

Villaverde, A. F., Bongard, S., Mauch, K., Müller, D., Balsa-Canto, E., Schmid, J., Banga, J. R., 2015a. A consensus approach for estimating the predictive accuracy of dynamic models in biology. Computer methods and programs in biomedicine 119 (1), 17-28.

Villaverde, A. F., Henriques, D., Smallbone, K., Bongard, S., Schmid, J., Cicin-Sain, D., Crombach, A., Saez-Rodriguez, J., Mauch, K., BalsaCanto, E., et al., 2015b. Biopredyn-bench: a suite of benchmark problems for dynamic modelling in systems biology. BMC Systems Biology 9 (1), 8.

Xu, P., Li, L., Zhang, F., Stephanopoulos, G., Koffas, M., 2014. Improving fatty acids production by engineering dynamic pathway regulation and metabolic control. Proceedings of the National Academy of Sciences 111 (31), 11299-11304.

Yinyu, Y., 1990. Interior-point algorithms for global optimization. Annals of Operations Research 25 (1), 59-73.

Zhang, F., Yi, X., Sun, X., Zhang, Y., 2006. [metabolism of recombinant cho-gs cell reducing of toxic effect of ammonia]. Sheng wu gong cheng xue bao= Chinese journal of biotechnology 22 (1), 94-100.

Zhuang, K., Yang, L., Cluett, W. R., Mahadevan, R., 2013. Dynamic strain scanning optimization: an efficient strain design strategy for balanced 
yield, titer, and productivity. dyssco strategy for strain design. BMC biotechnology 13 (1), 8. 


\section{Figure captions}

Figure 1. Methodology. The diagram shows the main steps of the method. Starting with a kinetic model, $n$ optimizations are performed in order to obtain a Pareto front of non-dominated solutions. From that set of solutions the one with the preferred trade-off between modification effort and performace improvement is selected. Finally, this solution is taken as the starting point for a final optimization (refinement) in which only a reduced subset of enzyme levels is allowed to change.

Figure 2. Metabolic network of the $\mathrm{CHO}$ cell model. It includes central carbon metabolism, amino acid metabolism, mitochondrial respiration, and generation of unwanted by-products.

Figure 3. Model calibration. Fits to the experimental data reported in (Nolan and Lee, 2011).

Figure 4. Pareto front. The blue dots represent non-dominated solutions, each of which presents an optimal trade-off between productivity (vertical axis) and $\epsilon$, the modification effort as defined in eq. (14) (horizontal axis). From this set of obtained solutions we selected the one corresponding to $\epsilon=2$, which presents the 4 significant modifications listed in the inserted text box.

Figure 5. Final solution. Comparison of the time-courses of the key variables of the original wild type (red lines) and optimized (blue lines) solution. Dotted lines represent upper bounds that are enforced as path constraints, i.e., conditions that must be fulfilled during all the time course of the experiment. Dashed lines represent lower bounds enforced as end-point constraints, i.e. they are minimum values that must be achieved at final time. 


\section{Tables}

Table 1: Enzyme levels: original and optimal values.

\begin{tabular}{cccc}
\hline $\begin{array}{c}\text { Enzymatic } \\
\text { modifications }\end{array}$ & $\begin{array}{c}\text { (I) Original } \\
\text { values }\end{array}$ & $\begin{array}{c}\text { (II) Pareto front } \\
\text { solution }\end{array}$ & $\begin{array}{c}\text { (III) Final refined } \\
\text { solution }\end{array}$ \\
\hline Glutamate dehydrogenase & 1.00 & 1.31 & 2.00 \\
Glutamate-ammonia ligase & 1.00 & 0.76 & 0.58 \\
Glucose phosphate isomerase & 1.00 & 0.30 & 0.26 \\
Slc25a18/Gc2 & 1.00 & 1.50 & 2.00 \\
\hline
\end{tabular}




\section{Figures}

Figure 1

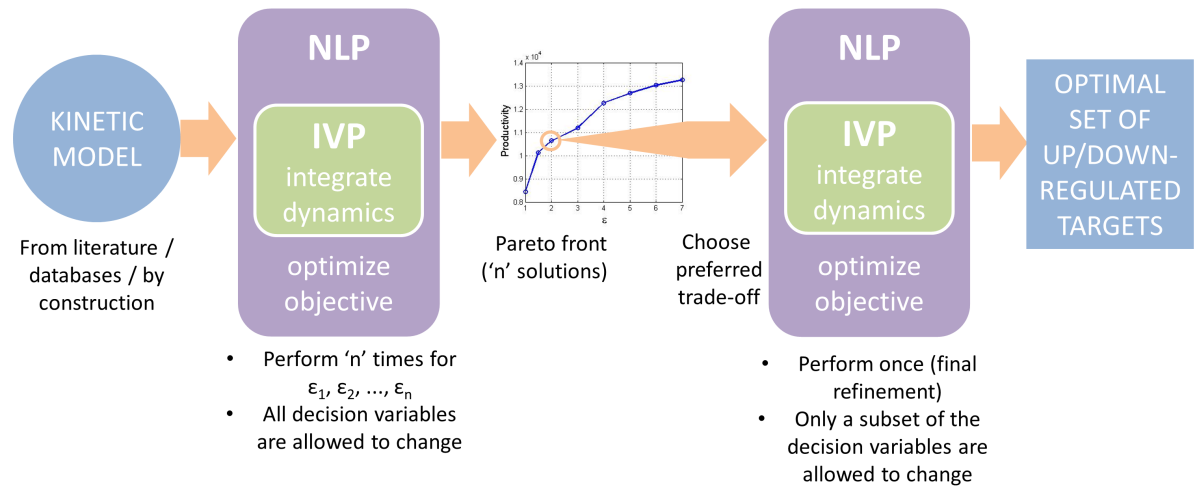


Figure 2

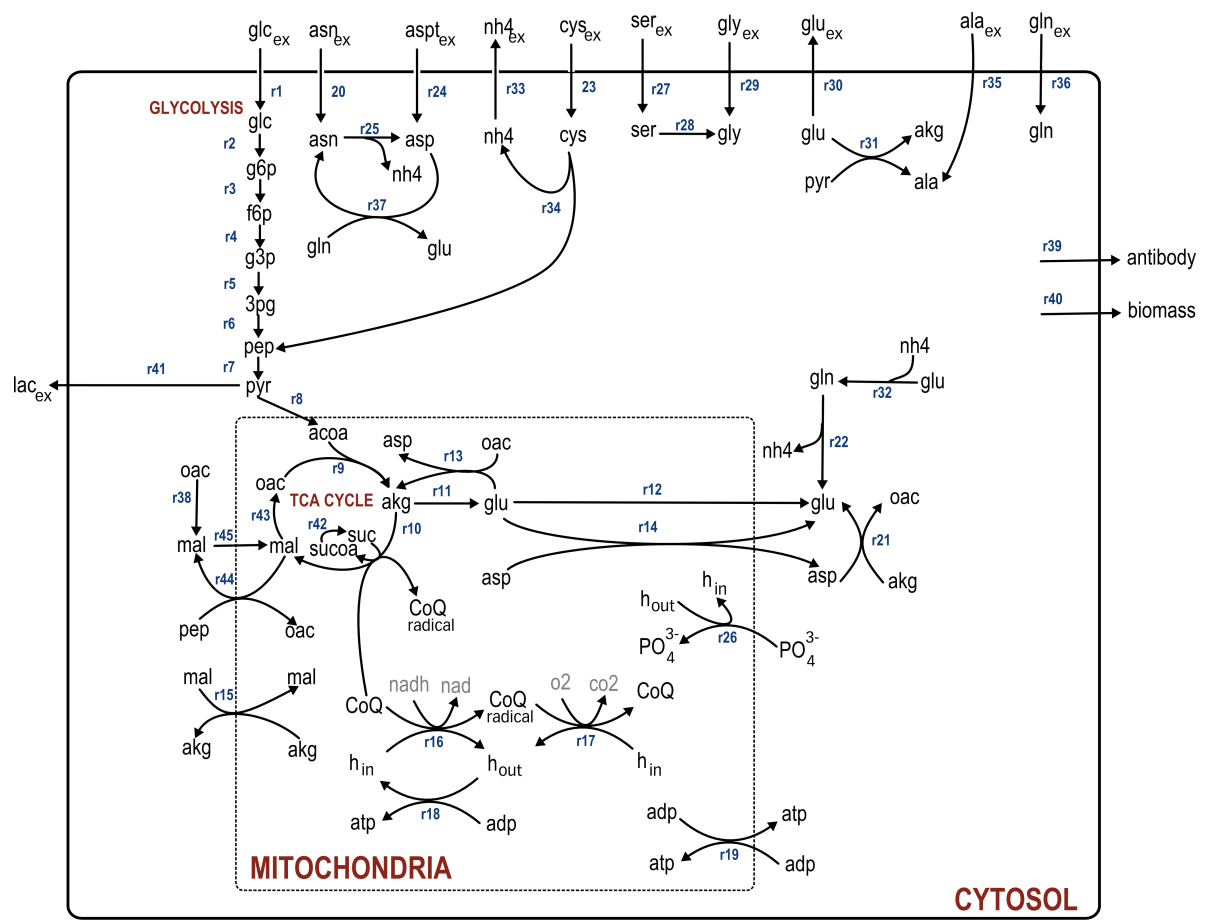

FERMENTER 
Figure 3

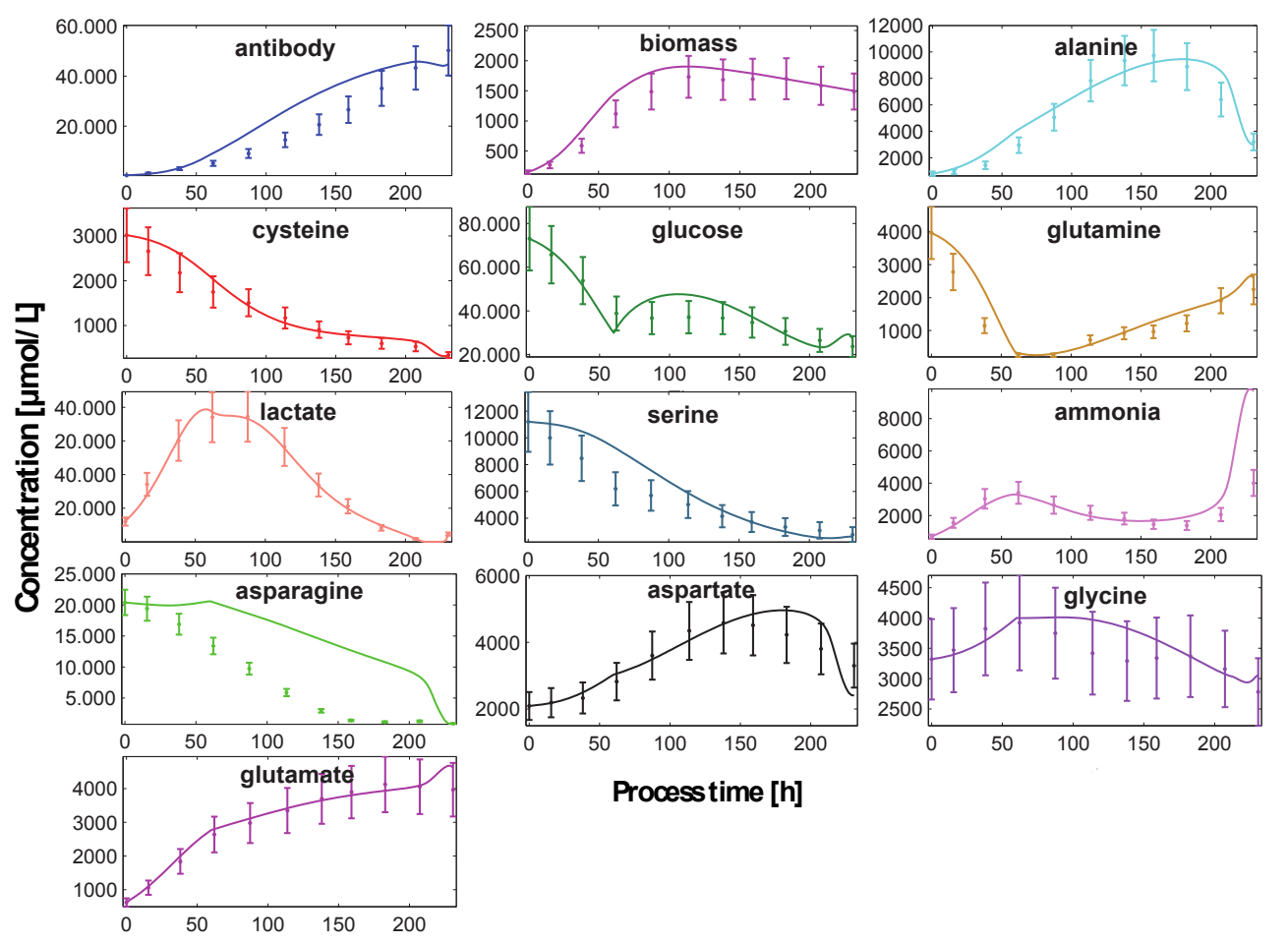


Figure 4

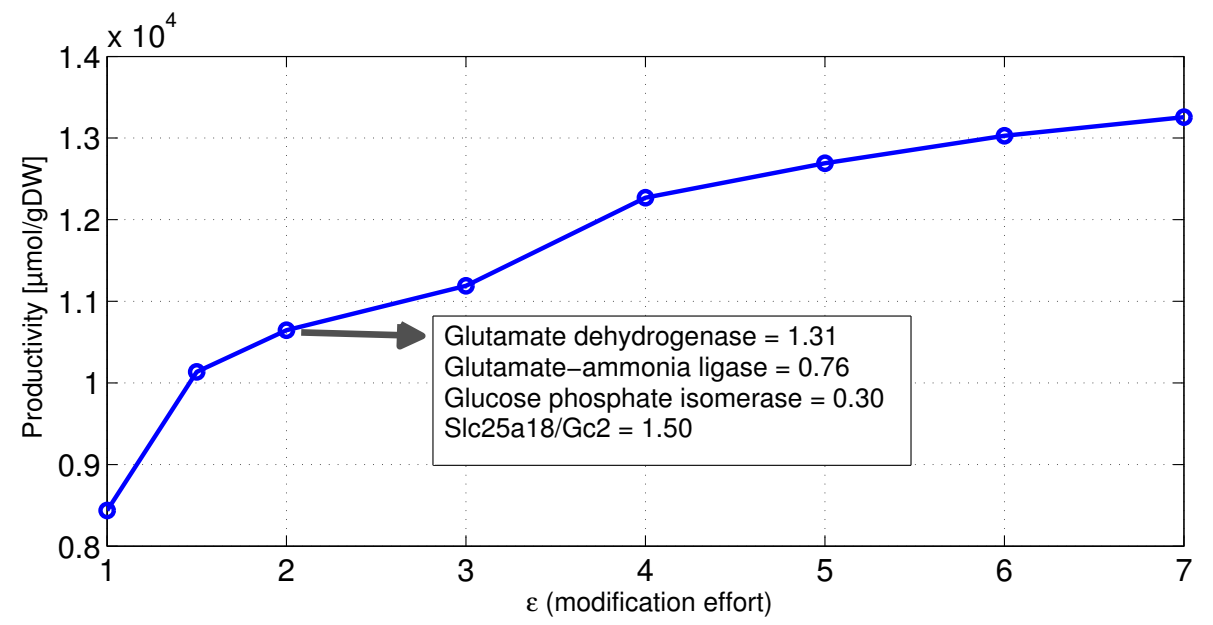


Figure 5

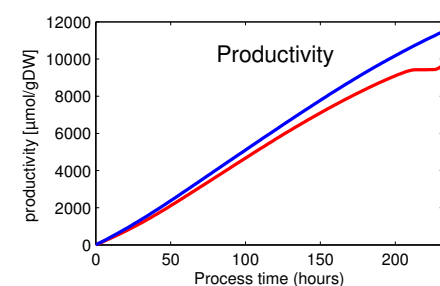

optimized lower bound (final time)

original upper bound (all time)
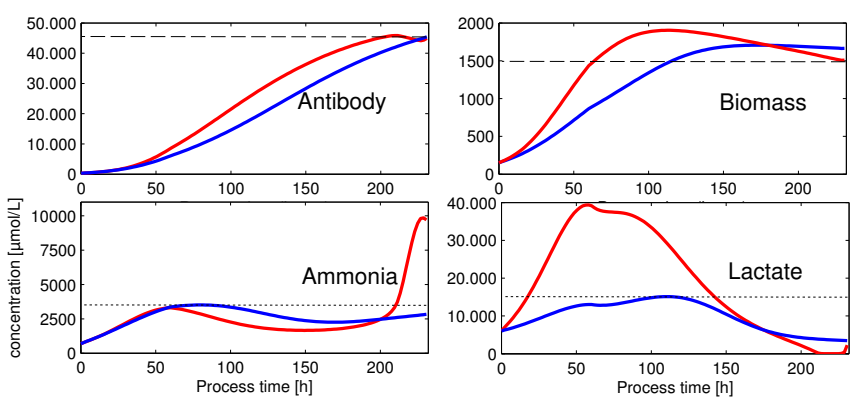